\title{
Linéaments politiques
}

En regard du frontispice du Léviathan

\section{Philippe Crignon}

\section{(2) OpenEdition}

\section{Journals}

Édition électronique

URL : http://journals.openedition.org/appareil/399

DOI : 10.4000/appareil.399

ISSN : 2101-0714

\section{Éditeur}

MSH Paris Nord

Référence électronique

Philippe Crignon, «Linéaments politiques », Appareil [En ligne], Articles, mis en ligne le 08 mai 2008, consulté le 30 juillet 2020. URL : http://journals.openedition.org/appareil/399 ; DOI : https://doi.org/ 10.4000/appareil.399

Ce document a été généré automatiquement le 30 juillet 2020

\section{(c) $(1)(9)$}

Appareil est mis à disposition selon les termes de la Licence Creative Commons Attribution - Pas d'Utilisation Commerciale - Pas de Modification 4.0 International. 


\title{
Linéaments politiques
}

\author{
En regard du frontispice du Léviathan
}

\section{Philippe Crignon}

1 Une philosophie de la culture, comme veut l'être la philosophie des appareils, se pose nécessairement la question de ce qui configure le commun; réflexivement, elle doit aussi s'interroger sur le lien qui est ainsi admis entre l'idée de configuration et l'idée de communauté :dans quelle mesure ce qui fait l'être-ensemble relève-t-il de la mise en forme, du dess(e)in ou de l'empreinte plutôt que, par exemple, du concept, de l'organisation ou du contrat; dans quelle mesure en retour la disposition du sensible engage-t-il le partage de la publicité jusque dans ses institutions politiques. Ces questions, courantes aujourd'hui, orientent le plus souvent les analyses vers une articulation de l'esthétique et du politique, visant, parmi d'autres notions, celle de "public" ou de "sensibilité commune" (pour ne rien dire des discours épuisés sur la soidisant nécessité d'un "art citoyen"). Notre propos ne sera pas ici d'évaluer directement de telles propositions, mais de tenter de dégager à quel niveau, dans la constitution du politique, il est exigé de parler en terme de figures ou de configurations. Nous entreprendrons cette recherche à partir de l'auteur avec lequel, aujourd'hui encore plus qu'auparavant, notre modernité politique tente de s'expliquer pour comprendre ses propres abîmes, échecs ou impasses, à savoir Hobbes.

2 Un tel choix s'impose également pour une autre raison: Hobbes nous a livré à la fois une théorie politique et une image, celle du Léviathan, image qui a pesé peut-être autant que le texte sur les commentaires ultérieurs, sans que soient toujours clairement identifiés l'écart et le rapport qui existent entre le frontispice et la doctrine. Cette image redouble et modifie les interrogations que suscite l'ouvrage. La relation qu'elle entretient avec le texte repose en partie sur un nom qui fait lui-même problème quant à son statut, celui de «Léviathan » qui est à la fois le titre de l'ouvrage, un certain nom propre de l'Etat hobbesien (thèse dont nous nous réservons la critique plus loin) et le colosse du frontispice. Le nom Léviathan revendique en lui-même l'unité de la figure, de la pensée et du politique ; que cette prétention soit un des problèmes fondamentaux que pose le livre, voilà ce que nous nous proposons de montrer. Il suffit de noter pour le moment qu'il est on ne peut plus probable que nous n'aurions pas la même entente de 
la pensée politique de Hobbes s'il n'y avait pas eu cette image. Ce n'est pas seulement qu'elle aura participé tant à l'enthousiasme qu'au rejet qui affecta et le livre et son auteur, mais ce sont aussi les concepts politiques décisifs - le corps politique, l'Etatpersonne, la représentation, la souveraineté - qui en auront été touchés.

C'est peu dire que l'ouvrage le plus accompli de Hobbes prit forme au contact de la réalité politique la plus intense de l'âge classique. Hobbes le rédige pendant son exil français en 1650, auprès de Charles II à qui il fera hommage d'un exemplaire manuscrit ; le livre associé à son image capitale ont, comme on sait, scandalisé très vite. Le Léviathan a toujours eu cet effet de hantise qui d'une part l'expose à toutes les accusations en sorcellerie (la diabolisation du hobbism est immédiate et récurrente) et qui d'autre part provoque des interprétations rigoureusement contradictoires (favorable à Cromwell/à Charles II, libéral/tyrannique, républicain/totalitaire etc. ; ce qui, du reste, induit à penser que la "thèse" politique de Hobbes n'est peut-être pas de l'ordre de la position). Il est aussi connu que c'est sous la figure du tyran absolu qu'a été d'abord repoussé le livre ; cette monstruosité de l'Etat (Leibniz : « Léviathan est un livre monstrueux comme son titre lui-même l'indique») se conjugue alors naturellement, semble-t-il, avec la monstration terrible du colosse dans le frontispice, figure du souverain-ogre qui incorpore les individus. L'excès politique que l'on attribue à la doctrine est aussi immédiatement un excès plastique. Cette dépendance d'une pensée et d'une image se traduit concrètement dans le fait que la plupart des éditions anciennes ou modernes du Léviathan se sentent comme obligées de reproduire la gravure inaugurale.

4 Attardons-nous alors sur cette image, attribuée à Abraham Bosse. On en connaît plusieurs versions dont l'une est un dessin (et non une gravure) qui figure en tête du manuscrit offert à Charles II. Les versions gravées, toutes similaires, sont plus nettes et comportent deux différences principales: le buste du colosse agrège des individus tournés vers la tête souveraine (et non de simples têtes tournées vers l'extérieur, ce qui lui donnait un aspect boursouflé), et la citation de Job («non est potestas super terram quae comparetur ») surtitre l'ensemble. Il faut ajouter que l'impact de cette image fut tel que Sorbière crut bon d'en adjoindre une copie - assez maladroite - à sa traduction française des Elements of Law-deuxième partie un an plus tard (sous le titre Le corps politique, qui n'est pas de Hobbes). A tout prendre, le frontispice est donc une condensation remarquable : un être gigantesque, un titre monstrueux et une citation sublime (il n'est rien à quoi on puisse le comparer).

Quant au rapport avec la théorie, il se lit d'abord de façon toute littérale comme figure de l'incorporation politique que décrirait Hobbes dans l'ouvrage. L'introduction du Léviathan livre en effet tout ce dont on a besoin pour justifier une longue série de correspondances entre l'homme naturel et le Grand Homme qu'est l'Etat. Deux voies s'offrent à l'analyse, commandées par deux figures que le frontispice superpose, selon d'ailleurs sa propre logique d'accumulation. Le première suit la figure du "corps politique", de la respublica comme organisme vivant, pensant et voulant. Le Léviathan fournira d'innombrables analogies entre le corps naturel et le corps artificiel de l'édifice politique. Toutefois, à la différence de sa présentation figurale, le "corps politique" chez Hobbes désigne l'Etat et non pas le seul souverain, qui n'en est pas même la tête mais l'âme ${ }^{1}$. L'écart n'est pas sans importance comme nous le verrons, mais déjà il indique quelles difficultés iconographiques poserait une illustration fidèle (comment représenter l'âme ?). La seconde voie réside dans l'image de la "personne 
artificielle" que développe le Léviathan dans son fameux chapitre XVI Des personnes. Le souverain est dit "porter la personne du peuple ", expression tout à fait traditionnelle dans le droit romain et médiéval (personam alicui gerere sive sustinere) pour désigner les universitates. A côté des personnes naturelles, douées d'une identité, de droits et de responsabilité, des collectivités peuvent se voir reconnaître le statut, fictif (mais bien réel en même temps; cette réalité de la fiction juridique est constitutive du droit romain et de ce qui l'a suivi ${ }^{2}$ ). Hobbes étend cette prétention à l'Etat mais, en même temps, il redéfinit radicalement la notion de personne, à partir de la représentation : une personne en général est un être intelligent qui parle et agit en son nom ou au nom de quelqu'un d'autre, qui se représente lui-même ou qui représente quelqu'un d'autre. Partant de là, le souverain devient le représentant du peuple, qui n'existe comme peuple que par le truchement de cette représentation (le peuple, de lui-même et par lui-même, n'est pas). Que ce soit donc sur le mode du corps ou sur celui de la personne, le frontispice paraît s'accorder à grands traits avec la conception hobbesienne de l'Etat.

6 A vrai dire, si le lien entre l'image et le discours s'en tenait à de telles correspondances, il relèverait de l'illustration la plus pauvre et la moins étonnante ; il serait parfaitement accessoire, bien loin de la nécessité que nous avons cru lui soupçonner et bien en-deçà de la puissance qu'il a assurément manifestée. Récemment, une autre interprétation est apparue pour rendre justice de ce qui se noue entre cette figure et cette doctrine, d'abord chez Noël Malcolm puis chez Horst Bredekamp ${ }^{3}$. L'un et l'autre mettent à jour à peu près les mêmes sources et les mêmes éléments pour souligner le rôle clé d'une technique, d'un instrument optique inventé dans les années 1620 et très à la mode dans les années 1640, soit au cœur de l'élaboration de la pensée politique de Hobbes. Cet instrument - un "perspectif" - est une lunette qui dispose d'un verre extérieur particulier, puisqu'il est polygonal. Face à cette lunette, fixée au sol, on tendait un écran sur lequel étaient dessinés divers portraits; celui qui regardait à travers le perspectif voyait une image reconstituée par les propriétés optiques du verre polygonal, image qui ne correspondait à aucune des figures effectivement dessinées sur le tableau mais qui empruntait à chacune un segment afin de composer un visage que seul l'instrument permet de révéler. Si aucun exemplaire n'a survécu, des témoignages existent sur ce dispositif, notamment chez le Frère Minime Nicéron qui lui a réservé une place de choix dans son ouvrage sur La Perspective curieuse (1638). Nicéron classe la lunette polygonale parmi les anamorphoses, ce qui lui permet de tenir un discours miludique, mi-sérieux sur la dialectique de la présence et de l'absence dans le visible, dialectique qui n'a plus qu'à se laisser investir d'une signification politique ou religieuse, c'est-à-dire d'une signification sur le pouvoir et sa manifestation :

«Le tout étant disposé de la sorte, il est certain que la peinture aura beaucoup plus de grâce et l'artifice en sera davantage estimé : mais encore plus si l'on se forme quelque dessein pour la signification de cette peinture, ce qui se peut remarquer en la 24 et $25^{\text {èmes }}$ planche és figures 69 et $72:$ ma première desquelles est à peu près la copie au moins le dessein d'un tableau que je traçai et fis peindre il y a 2 ou 3 ans et qui se regarde encore à présent en la Bibliothèque de notre Couvent de la place Royale à Paris. Ce tableau dressé en la façon, que nous avons dit en ce livre, étant vu directement, représente une quinzaine d'Ottomans vêtus à la Turque, la plupart tirés d'un livre intitulé Icones Sultanorum : et quand on vient à regarder par la lunette, au lieu de ces Ottomans, on ne voit plus que le portrait de sa Majesté TrèsChrétienne très bien fait, ressemblant et vêtu à la française, encore qu'il se compose de plusieurs pièces des autres portraits, qui se ramassent et s'unissent ensemble pour le former tel qu'il se voit. » 
«Ce dessein est fait à propos de la Prophétie au moins tenue pour telle, par ceux à qui elle a été donnée que Mahomet laissa autrefois à ses successeurs. Leur recommandant de ne jamais offenser la Monarchie française, parce que leur empire ne serait jamais ruiné que par la puissance de quelqu'un de ses rois. Sur ce voulant montrer que l'honneur de cette conquête n'appartient point à d'autre qu'à Louis le Juste, nous faisons que la plupart de ces empereurs en ce tableau lui rendent hommage, en sorte qu'ils contribuent chacun quelque partie de soi pour former son image, comme s'ils se dépouillaient eux-mêmes pour honorer son triomphe ${ }^{4}$

7 C'est un lieu commun de rappeler que les anamorphoses mettent en jeu, le plus souvent, le portrait des grands; Nicéron propose dans les planches qu'il destine au verre polyoptrique des exercices sur celui de Louis XIII et sur celui d'Urbain VIII, soit le souverain politique et le souverain pontife. Comme beaucoup de perspecteurs, Nicéron manifeste à la fois une réelle foi mystique dans ces instruments et leur puissance révélatrice (Maignan compare de son côté la perspective à la transfiguration eucharistique) et une grande fantaisie qui démine toujours assez tôt le trop de sérieux qui pourrait s'y attacher. Le miracle qui fait qu'un souverain réside invisiblement en tous ses sujets tout en se révélant visiblement comme l'agglomération des individus, est toujours un miracle technique.

8 La potentialité symbolique de cet instrument optique n'a pas échappé à Hobbes, dont Malcolm et Bredekamp rappellent qu'il est justement l'auteur de l'une des deux autres sources littéraires qui témoignent à l'époque de l'existence et de la diffusion de cette anamorphose. Le texte de Hobbes est une réponse à la préface du Gondibert, poème de Davenant. Après avoir rappelé la portée politique de ce poème, qui décrit l'affrontement entre la voie de la pacification politique et la voie de la discorde factieuse (entre la paix et la guerre, l'Etat et l'anarchie), Hobbes emploie la métaphore du verre polyoptrique :

«Je crois, Sir, que vous avez vu une perspective curieuse où celui qui regarde à travers une courte lunette vers une image qui contient diverses figures n'en voit aucune d'entre celles qui sont peintes, mais quelque personne composée de segments, convoyés vers l'œil à travers un verre taillé ingénieusement ?artificiall? »

9 Il ne fait aucun doute que dans ces lignes, Hobbes renvoie la compréhension de ce qu'est l'autorité souveraine au perspectif décrit plus haut. Tout concourt à assurer que Hobbes avait connaissance de celui-ci : l'amitié avec Mersenne auquel il rendait visite au Couvent de la Place Royale dans les années 1634-1636, c'est-à-dire au moment où Nicéron y peignait son anamorphose, son intérêt pour les questions optiques, dans un dialogue suivi avec le même Mersenne (qui joue ici le rôle d'intermédiaire : il a eu la charge d'accorder le privilège de la congrégation au livre de Nicéron et il a publié le Tractatus Opticus de Hobbes) et enfin le fait que Hobbes cite La Perspective curieuse dans le De Homine de 1658, à propos, certes, d'une autre anamorphose que celle du verre polyoptrique. Il est donc pertinent de rapprocher un dispositif optique qui réalise le miracle d'une union d'individus incarnée dans une seule personne souveraine et la procédure qui unit les hommes atomisés sous l'autorité d'un pouvoir qui les incorpore. Le nœud se resserre définitivement lorsqu'on établit l'identité du corps optique et du corps politique, ce que fait une dernière source littéraire, celle de Fanshawe, dans un texte qui plus est dédié à Charles II en 1648 :

«Votre altesse a peut-être vu à Paris une image (elle se trouve dans le cabinet du grand chancelier) qui est si admirablement imaginée qu'elle présente à

l'observateur ordinaire une multitude de petits visages (les glorieux ancêtres de ce 
gentilhomme), mais en même temps, celui qui regarde à travers un perspectif disposé là à cet effet voit un seul portrait en grand format du chancelier lui-même. Par là, le peintre donne à entendre qu'en la personne du chancelier se concentrent toutes les vertus de ses ancêtres; ou, par une philosophie plus subtile, il parvient à montrer que le corps politique est composé d'un grand nombre de corps naturels et que chacun d'entre eux, en soi complet et consistant en une tête, des yeux, des mains et pareilles choses, est, dans l'autre, une tête, un œil ou une main ; et qui plus est, que les corps privés des humains ne peuvent être préservés si le corps public est détruit, tout aussi peu que ces petites images ne pourraient conserver leur existence si la grande était corrompue; car cette grande image était aussi la première et la plus importante dans le dessin du peintre, et c'est en son nom que tout le reste a été fait. $»^{6}$

10 Avec ce texte se conclut une série d'analyses tendant à superposer le frontispice et la doctrine politique; il est, pour Bredekamp «le dernier maillon d'une chaîne démonstrative appuyant la thèse que le Léviathan est, pour Hobbes, le produit d'un verre perspectif $»^{7}$. Cette démonstration pourtant est presque trop belle, trop esthétique et harmonieuse ; elle est une œuvre d'art à soi seul, où chaque élément s'emboîte parfaitement ${ }^{8}$. Elle incorpore elle-même l'ensemble des indices dans un tout où chaque parcelle prend sens. Que cette herméneutique ait la forme de l'intégration totale, voilà qui, au lieu de susciter l'admiration, doit faire question, et il faudrait se demander si 1) elle ne détermine pas ses propres résultats, la splendide identification $\mathrm{du}$ corps du frontispice et du corps politique, leur incorporation à un niveau supérieur, et si 2) elle ne conditionne pas les données du problème : le Léviathan identifié à l'Etat hobbesien, lui-même identifié au corps politique. Ce sont là des présupposés (qui tous reposent sur l'identification ou la condensation) que nous allons examiner, non seulement parce qu'il importe d'éclaircir la pensée politique de Hobbes au vu des préoccupations contemporaines, mais également parce qu'une telle interprétation n'est pas sans charge mythologique, et cela concerne autant le dispositif optique et le corpus politicum que le projet ou la portée d'une "iconographie politique" en général. Pour poursuivre nous essaierons de répondre à ces questions : le corps est-il une image du politique chez Hobbes? l'union politique se fonde-t-elle sur un dispositif et si oui lequel ? l'Etat est-il identifiable au Léviathan?

11 La comparaison entre la société politique et le corps n'a pas attendu Hobbes et se retrouve dans les textes les plus divers et les cultures les plus lointaines, encore faut-il distinguer entre un usage strictement métaphorique et rhétorique et un emploi plus fondamental où le corps détermine le politique en son essence. Lorsque Platon pose l'analogie entre les politeiai et les constitutions physiologiques pour évoquer les maladies politiques, le lien demeure extérieur entre les deux termes de la comparaison'. L'antiquité pré-chrétienne ne pense jamais - sinon de manière métaphorique - le politique comme $\operatorname{corps}^{10}$ (concept qui exigerait lui-même des contours plus précis et des différenciations en organisme, corps humain, corps médical, automate, etc.) et ce n'est pas avant Saint Paul que se met en place une formule à la fois précise et prégnante du corps communautaire. Si la donne change avec Saint Paul, c'est essentiellement parce que le christianisme a adopté le corps comme pivot (l'Incarnation) tout en reconfigurant totalement la signification de ce qu'est un corps (le corps est chair). Sans ces deux modifications historiques, le corps n'aurait jamais pu définir l'essence de tout pouvoir légitime ni engendrer une théologie politique séculaire $^{11}$. Saint Paul a défini l'Eglise comme corps dont la tête est le Christ et dont les membres sont les fidèles. La foi définit le lien d'adhésion à ce tout dont on reconnaît 
n'être qu'une partie. Mais ce grand corps est un corps marqué par la corruption et destiné à la rédemption : il revient à l'Eglise d'accomplir l'économie divine. Deux voies prolongent cette "matrice" paulinienne. La première suit la ligne que dessine le corps : être fidèle, c'est s'incorporer au corps de l'Eglise; cela s'institue par le baptême et se perpétue dans le rituel eucharistique (où, réciproquement, le corps christique est incorporé à celui du fidèle). La seconde suit la ligne iconique : être fidèle, c'est imiter Paul qui imite le Christ, lui-même "première image", image du Père. On ne peut pas dissocier les deux voies : le corps en tant que chair est essentiellement un corps qui se rend visible, il immisce l'invisible dans le visible et noue la transcendance à un déploiement des apparences. Inversement, l'image incarne, s'anime et touche. Chair et image se conjuguent et vivent au même rythme: le christianisme obéit à un mouvement de perte et de reprise ; c'est par la chair que le péché est venu, c'est par l'Incarnation que le péché est rédimé, c'est par l'image-idole que l'homme a tourné le dos au monothéisme, c'est par l'image-icône qu'il renoue avec lui, c'est par le pouvoir, enfin, que la corruption s'est imposée, c'est par le pouvoir de l'institution ecclésiastique que la corruption sera chassée.

On voit donc que le lien qui unit le corps et l'institution n'a rien de rhétorique ou de pédagogique. Il ne nous revient pas ici de reprendre les analyses majeures de Kantorowicz sur l'évolution du concept. Il suffit de rappeler que l'Eglise - corpus Christi uni au Pape, caput Ecclesiae - s'est impérialisée pour devenir ce corpus mysticum ecclesiasticae qui servit en retour de modèle (selon un mouvement de balancier bien connu) à l'institution politique impériale, sous l'expression mimétique de corpus mysticum reipublicae au XIII ${ }^{\mathrm{e}}$ siècle. La formule de corpus politicum qui désigne d'abord l' autre corps du roi (le corps non privé, non naturel; le corps infusé de l'autorité politique reçue - de loin en loin - de Dieu), s'appliquera plus tardivement à l'ensemble de l'Etat. La conception du corps politique s'est ainsi complètement accomplie avant Hobbes (le livre de Kantorowicz s'arrête significativement aux Tudors). Et si nous nous sommes permis de rappeler ces points essentiels de la théologie politique, c'est pour faire remarquer une chose simple: alors que la doctrine du corpus politicum a déployé ses possibilités depuis bien longtemps déjà à l'époque de Hobbes, aucune illustration ou figuration n'en existe avant le frontispice du Léviathan. Ne pas s'interroger sur ce paradoxe chronologique expose inévitablement à des mésinterprétations principielles. Jamais pendant les siècles où il opérait avec le plus d'acuité, le concept de "corps politique" n'a fait l'objet d'une image ou d'une représentation, et cela alors même que la matrice paulinienne dans laquelle il prend sens a des engagements picturaux précis (l'icône, le retable, le cérémonial par exemple). Il faut sans doute partir de ce problème et se demander si le frontispice de 1651 a seulement à voir avec une théorie du corpus politicum.

13 Hobbes semble à bien des égards suivre la tradition incarnationnelle de l'Etat et il est certain qu'il emploie à diverses reprises le concept de "corps politique". Toutefois, un examen des textes de Hobbes doit tenir compte de son évolution interne, depuis les Elements of Law (1640), le De Cive (1642) jusqu'au Léviathan (1651), évolution qui correspond à la recherche proprement hobbesienne d'une solution du politique. On ne saurait donc reconnaître la pensée politique de Hobbes dans son seul point de départ, puisque celle-ci est avant tout une force polarisée vers son point de légitimité, qui ne se découvre que dans le dernier ouvrage. Une telle précaution s'impose d'autant plus pour notre propos qu'elle révèle une évolution sensible du concept corporatiste. Dans les Elements de la loi naturelle et politique, Hobbes pense l'union politique en termes 
d'intégration des volontés particulières dans une seule et de transfert de droits; cela constitue, selon lui, « un corps politique - ou société civile - et les Grecs l'appellent (...) une cité, qui peut être définie comme étant une multitude d'hommes, unis en une seule personne. $»^{12}$ Nous avons ailleurs ${ }^{13}$ montré l'insuffisance de cette première tentative : fonder l'Etat sur un contrat au sens d'un transfert juridique ne permet pas de rendre compte de la puissance de l'Etat. Outre qu'un contrat suppose une garantie institutionnelle et qu'il ne peut donc pas ériger lui-même l'édifice dont il a préalablement besoin, la procédure transactionnelle ne peut obliger qu'à ne pas résister à la force du souverain. Celui-ci serait sans doute sans obstacle mais ne serait pas plus puissant pour autant ; la constitution du pouvoir politique est ainsi ratée. C'est pourquoi les expressions fréquentes de "corps politique" et de "personne juridique" appliquées à l'Etat en 1640 sont de portée limitée et pour tout dire rhétoriques. Hobbes emploie ici une terminologie qui n'est pas la sienne, parce qu'il n'a pas encore inventé sa propre langue, comme le montre encore par exemple l'équivalence traditionnelle qu'il maintient entre l'Etat et la "société civile" (civitas sive societas civilis).

Le De Cive constitue l'étape intermédiaire dans le parcours de Hobbes et se signale à trois titres. Tout d'abord, la notion de "corps politique" disparait complètement et littéralement du texte, du moins du texte latin et de la traduction anglaise (Sorbière, quant à lui, rétablira régulièrement l'expression dans sa traduction en 1649). Absence significative, puisque l'Etat demeure tout de même une "persona civilis"; c'est donc bien le schème de l'incorporation qui est congédié ici. En second lieu, Hobbes s'explique sur cette absence dans un paragraphe qui déclare possible (donc non nécessaire, et c'est déjà suffisant pour rompre avec la pensée théologico-politique) la comparaison corporatiste à condition de l'amputer d'un trait essentiel : le souverain ne doit pas être comparé à la tête mais à l'âme de l'Etat (VI, xix). Soit on considère que la différence est mince et on ne rend pas compte de l'absence de la métaphore dans l'ouvrage ni du paragraphe en question, soit on lui accorde une signification et une portée qui ne peut être que celle-ci : l'âme est séparée du corps qu'elle gouverne, au contraire de la tête qui est une partie intégrante de ce corps; la distinction du corps et de l'esprit valant même pour le matérialisme de Hobbes ${ }^{14}$. Ce qu'esquisse donc ici Hobbes, ce n'est donc pas l'incorporation mais bien au contraire la séparation de l'Etat et du peuple, dont l'union n'est cependant pas encore fondée. Ceci nous conduit à la troisième innovation du texte de 1642: afin d'empêcher toute résurgence de la pensée incarnationnelle, Hobbes développe une critique décisive du corpus mysticum ecclesiae dans la troisième section. Ce qui constitue donc la racine historique et ontologique du "corps politique" est totalement évidé. L'alternative qu'impose Hobbes ne laisse aucune chance de survie à l'Eglise comme "corps mystique". Soit on entend par Eglise une communauté concrète, capable de se réunir sous l'autorité d'un souverain (et alors l'Eglise n'est rien d'autre qu'un Etat chrétien), soit on entend par Eglise une communauté invisible et universelle, qui n'a alors aucune réalité effective :

«L'Eglise universelle est en effet un corps mystique dont le Christ est la tête, mais pas autrement que tous les hommes qui, ensemble, reconnaissent Dieu comme étant le Souverain du monde, ne composent un seul Royaume et une seule Cité, sans être pour autant une seule Personne, dotée d'une action commune ou d'une intention commune (...) Bien plus, là où le Christ est dit tête du corps de l'Eglise, l'Apôtre l'a manifestement entendu des Elus qui, aussi longtemps qu'ils sont de ce monde, ne forment une Eglise qu'en puissance ${ }^{15}$ 
L'Eglise universelle, distincte de l'Etat, devra donc attendre l'au-delà pour avoir quelque réalité ; d'ici là, elle ne peut être que particulière, nationale et politique, sans clergé distinct des offices juridiques relevant du souverain. Cette position "érastienne" se conjugue donc avec une liquidation en règle du corpus mysticum et de toutes les constructions politiques qui s'en sont nourries, ce qui explique la disparition du schème corporatiste pour l'Etat dans le De Cive.

$\mathrm{Au}$ terme de ce parcours, le Léviathan apparaît comme un cas complexe. Sans les investigations précédentes, on risquerait de ne pas s'interroger sur un "certain" retour du schéma macrocosmique de l'Etat, encore faut-il d'une part être précis, et d'autre part pouvoir problématiser ce "retour". Pour la précision, tout d'abord, il faut noter que Hobbes compare, quand il le fait, l'Etat à un grand homme, un homme artificiel et non pas à un grand corps. L'introduction au Léviathan tisse la métaphore déjà assez loin, l'ouvrage détaillera une série de correspondances avec une ironie croissante: Hobbes évoque les articulations de l'Etat (les magistrats), ses muscles (les associations civiles), ses abcès (les groupes illicites), ses nerfs et ses tendons (les ministres), ses veines (les trésoriers), ses malformations, son hydrophobie, son épilepsie, ses fièvres, sa pleurésie, ses kystes, etc. Il suffit de lire la seconde partie pour constater que ces comparaisons surgissent toujours à la fin d'analyses qui ne leur doivent théoriquement rien du tout. Toujours pour être précis, il faut noter encore deux choses. En premier lieu, la comparaison anthropologique (et non plus corporatiste ${ }^{16}$ ) repose elle-même sur une comparaison mécaniste. C'est ce que dit l'introduction : la techné peut imiter la nature (l'homme peut fabriquer un "animal artificiel") parce que celle-ci est elle-même une techné (« qu'est-ce que le cœur sinon un ressort (...) ?»); si donc les hommes peuvent instituer ce "grand homme" qu'est l'Etat, c'est parce que l'homme est déjà un artefact naturel, produit par un Dieu ingénieur. Hors de l'horizon mécaniste qui enchaîne techné et phusis, la métaphore anthropologique n'aurait plus de sens. Enfin, dernière remarque: la locution de "corps politique" qui apparaît vingt-quatre fois dans l'ouvrage désigne toujours, à deux exceptions près dont l'une se trouve dans l'introduction ${ }^{17}$, les groupes subordonnés et non pas l'Etat. Il n'y a donc pas de retour en arrière avec le Léviathan, mais une manière plus complexe de concevoir l'Etat. On ne saurait donc se rendre aveugle à cette complexité et aligner la pensée de Hobbes sur la doctrine corporatiste dont il est au contraire le fossoyeur. Ce qui reste à interroger, c'est finalement la portée d'un frontispice qui figure à sa manière une certaine incorporation qu'on ne trouve nulle part dans le texte. C'est l'écart entre l'image et la doctrine qui est frappante maintenant, et non pas leur proximité.

Pour mener à bien cette analyse, il faut revenir à ce qu'il y a de neuf dans le Léviathan et qui le sépare des ouvrages de la décennie précédente. Le Léviathan est le seul des trois traités à réaliser l'ambition de fonder l'Etat (et de fonder la science de l'Etat par la même occasion, comme le rappelle Yves Charles Zarka). Les Elements of Law et le De Cive s'en tenaient à un impossible contrat social et politique. Or tout contrat est juridique et le droit suppose l'autorité politique. L'origine de l'Etat au sens de sa condition de production reste donc à chercher ailleurs que dans une procédure. Enoncée dans le chapitre XVI, la théorie de la représentation constitue l'apport décisif de Hobbes, elle est ce qui donne au politique une détermination et une configuration. Le souverain représente le peuple et celui-ci n'existe que parce qu'il est représenté. Il ne s'agit donc pas (ou pas fondamentalement) de transférer des droits mais de conférer une autorité, c'est-à-dire d'instituer le pouvoir tout en se reconnaissant les auteurs individuels et 
collectifs des actes du souverains. La représentation est la modalité par laquelle le peuple est et n'est pas le souverain. D'une certaine manière tout ce que décide celui-ci est, par principe, décidé par le peuple en vertu de l'autorité investie : « chaque sujet est, en vertu de cette institution, l'auteur de toutes les actions et de tous les jugements du souverain institué $»^{18}$. Mais en même temps, le peuple a livré (terme qui cherche à éviter ceux d'aliénation et de délégation, tous deux trop immobilisés dans ce mouvement du Même et de l'Autre) tous les pouvoirs au souverain qui n'a par définition de compte à rendre à personne. Représenter possibilise l'identité et la différence. C'est à une profondeur qui est celle de l'ontologie qu'il faut ainsi envisager la nouveauté du Léviathan; cela veut dire aussi bien qu'il atteint l'essence du politique (et non de la politique, entendue dans ses thématisations et ses pratiques) qu'il range le politique comme une nécessité de l'ontologie (l'être en tant qu'être a une portée politique). La représentation dessine une nouvelle configuration onto-politique, et cela parce que Hobbes l'a absolutisée ${ }^{19}$ (au contraire donc de toutes les procédures juridiques de représentation au sens de la délégation, qui est essentiellement finie, limitée quant à ses termes : un délégué n'a jamais les pleins pouvoirs). L'acte historique effectué par Hobbes doit être précisé sur certains points essentiels :

a) La représentation n'est pas seulement un dispositif de reflet de la volonté du peuple, elle constitue le peuple qui n'est rien sans elle, sinon une multitude atomisée. La représentation absolue étant posée, il ne peut rien exister avant elle (il n'y a pas d'appartenance préalable à la famille, aux métiers, aux cités, etc.), pas même cet «état de nature» dont Hobbes aura été le curieux inventeur puisque cette situation prépolitique n'est ni un état au sens de la stabilité (elle est l'inquiétude permanente pour la vie) ni conforme à la nature (la nature elle-même est détruite ${ }^{20}$ ).

b) La représentation instaure l'égalité politique de tous les individus car la souveraineté ne se partage ni ne se parcellise. Le souverain a le monopole de l'inégalité. La citoyenneté sous la représentation est un statut horizontal.

c) Elle évite deux solutions historiques connues. D'une part elle se passe de la participation immédiate de chacun aux affaires politiques (solution grecque). D'autre part elle n'intègre pas les individus à un dessein providentiel et à une économie finalisée par le salut (solution théologico-politique). La loi du souverain est la loi de tous et de chacun (au double sens du génitif), mais en même temps, chacun est dispensé d'y collaborer. Le mode onto-politique de la représentation est celui de la séparation. Celle-ci n'est pas à entendre comme l'absence de rapport mais comme un mode spécifique de rapport; cette séparation constitue la "société civile" distincte de l'organe de l'Etat. L'expression de civitas seu societas civilis qui valait encore pour les deux premiers traités est devenue impossible en 1651 : désormais, lorsque Hobbes veut dire Etat il dit Etat (commonwealth, civitas, respublica) et lorsqu'il veut parler des relations interhumaines pacifiées grâce à l'Etat il dit société civile (au sens même parfois de civilité et de courtoisie).

21 d) La représentation possède dès son origine les traits essentiels de la politique moderne : elle laisse chacun s'occuper de ses propres affaires privées (les hommes ne font plus corps) et réalise le désintérêt connexe des individus pour la politique. Celui-ci n'est donc pas en lui-même un risque mais une donnée fondamentale.

22 La politique n'est pas une simple région des affaires humaines, elle s'articule d'ellemême, c'est-à-dire depuis son essence configurée, à une métaphysique. La représentation politique suppose la souveraineté absolue (ab-solue c'est-à-dire 
séparée) ; pourtant elle rencontre - et sans que soit remise en cause son illimitation propre une autre sphère tout aussi absolue, qui est celle de la conscience. Il n'y a pas un simple parallèle entre la politique de la représentation et la métaphysique de la conscience ; c'est depuis l'institution de la souveraineté représentante que doit se poser celle de la conscience. Hobbes est le premier à dire clairement (et cela pour des raisons non pas morales mais structurelles), que la politique ne commande que les actes, qu'elle n'implique donc aucune adhésion du cœur, in foro interno, à la loi. Cette désimplication de la conscience achève sans doute de ruiner l'idée d'une théorie du "corps politique" chez Hobbes, à laquelle correspondrait le frontispice. Nous réservons pour plus tard la question que celui-ci pose, question qui ne peut être problématisée qu'à partir de la mise en évidence de l'écart entre l'image et le texte. Tâchons d'abord d'expliciter l'articulation entre souveraineté absolue et liberté de conscience. Il faut immédiatement préciser qu'il ne s'agit pas d'une limitation du pouvoir politique, au contraire : le souverain serait fragilisé si les hommes devaient, par principe, adhérer à loi qui les obligent. Hobbes classe ce préjugé au rang des germes de guerre civile. Si l'on attend (que cette attente soit parfois déçue est une autre histoire) l'approbation de chacun aux actes qui sont exigés par le pouvoir politique, il faut alors configurer celuici à partir d'une incorporation, d'une foi et d'une fidélité, ce qu'a précisément réalisé le théologico-politique. Hobbes décide tout autre chose: la représentation politique, la désincorporation nécessaire de l'Etat, l'obligation d'agir selon la loi et la liberté de croire ce que l'on veut. Telle est par exemple la distinction que fait Hobbes entre la confession (publique) et la foi (intérieure). La reconnaissance de la souveraineté absolue de la conscience est donc une nécessité pour la souveraineté absolue du pouvoir politique. La métaphysique de la conscience, ou la métaphysique de la représentation (au sens idéel) est intimement nouée à la politique de la représentation. $L a$ représentation se délivre maintenant en son unité essentielle, comme la figure métaphysique et politique de la modernité.

L'unité de la représentation n'est pas que nominale; elle a la forme de la séparation dans l'un et l'autre champ. La représentation mentale sépare le monde extérieur de l'idée que je peux m'en faire dans le domaine souverain de ma conscience, sachant que je ne dois pas attribuer à l'objet ce qui n'appartient qu'à sa représentation (couleurs, odeur, etc.). De ce point de vue, l'attitude de Heidegger qui désigne Descartes comme le fondateur de l'époque de la représentation est symptomatique d'un double déni : de la part réservable à Hobbes dans la fondation de cette époque et de la portée proprement politique de la représentation (y compris sans doute chez Descartes). Le trait de la séparation si soigneusement analysé chez Descartes dans le passage lorsqu'il met en doute l'existence du monde extérieur (à laquelle survit sa représentation), peut tout aussi bien s'observer chez Hobbes comme l'a magistralement démontré Yves Charles Zarka $^{21}$, dans le thème de l'annihilatio mundi :

«Pour la compréhension de ce que je veux dire par la puissance cognitive, nous devons nous rappeler et nous devons reconnaître qu'il y a continuellement dans nos esprits certaines images ou conceptions des choses à l'extérieur de nous, à tel point que si un homme pouvait être vivant et que tout le reste du monde fût annihilé, il en conserverait néanmoins l'image, ainsi que l'image de toutes ces choses qu'il avait auparavant vues et perçues en lui, chacun, par sa propre expérience, sachant que l'absence ou la destruction des choses imaginées à un moment donné ne cause pas l'absence ou la destruction de l'imagination elle-même. Ces images ou représentations des qualités des choses à l'extérieur de nous sont ce 
que nous appelons notre "cognition", notre "imagination", nos "idées", notre

"notion", notre "conception" ou notre "connaissance" des qualités des choses. ${ }^{22}$

L'élaboration de la représentation mentale en tant que détermination de l'être - selon laquelle, comme dit Heidegger, cogitare = percipere - connaît, chez Hobbes comme chez Descartes, un accompagnement dans la science de l'optique. Hobbes s'engage à la suite de Képler et développe une théorie de la vision qui se nourrit, à partir de 1637, de sa polémique avec Descartes. Les traités scientifiques ${ }^{23}$ suivront une ligne parallèle aux traités politiques; il ne convient pas de forcer les liens entre les deux champs de recherche, l'apport de Hobbes à l'optique demeurant en-deçà de son importance dans la pensée onto-politique. Mais son évolution scientifique n'est pas étrangère à l'instauration époquale de la représentation, et elle concerne deux points. Alors qu'en 1646, Hobbes fait de l'optique la science de la vision et étudie ses deux moments physiologique et psychologique, il recirconscrit l'optique autour de la formation des représentations au sein de la conscience dans le De Homine (1658). Isolée et reconduite dans le domaine qui est le sien, la représentation n'est plus censée prolonger l'événement physique oculaire ; une incision est tracée entre le moment cérébral et le moment mental et la question de la ressemblance entre les picturae rétiniennes et les imagines mentis devient inessentielle. En outre, Hobbes passe progressivement d'une pensée de la représentation comme affection à une pensée de la représentation comme acte de la conscience (fancy, phantasma, apparence, representation). Si l'intérêt de Hobbes pour l'optique peut résonner avec la mise en place bien plus large de la politique de la représentation, ce n'est pas au moyen d'un dispositif technique comme la lunette polyoptrique que le lien se noue, mais par la délimitation de la région conscience.

Ce qui est mis en évidence à ce stade, c'est une unité de la représentation en ses différentes acceptions (optique, picturale, métaphysique, politique), l'équivocité du mot n'étant pas nécessairement à mettre sur le compte d'un brouillard conceptuel, même s'il est tentant de renvoyer cette unité à un phénomène purement langagier. On ne peut pas s'en débarrasser à si peu de frais car son cas n'est peut-être pas si différent de celui de l'être dont Aristote répétait qu'il se dit pollakôs, de diverses façons. Refuser de penser cette unité ne serait légitime qu'à la condition de conceptualiser chaque signification singulière et d'en montrer la différence d'essence avec les autres significations, ce qui nous semble-t-il n'a jamais été fait (sur le mode de l'évidence : il est évident qu'il s'agit d'autre chose). Sans que nous puissions ici faire plus qu'esquisser la démonstration du contraire, nous pensons que peut être établie cette unité de la représentation à condition de la saisir en ce qu'elle est fondamentalement. La voie qui s'ouvre le plus facilement pour rejoindre cette unité consiste à suivre l'œuvre de la représentation, ce qu'elle produit, ce qu'elle affecte. Et cela, nous avons pu déjà le relever, consiste à parcourir ce que trace la représentation, à savoir une séparation. Le rapport au monde comme à la politique se constitue comme séparation, qui est, répétons-le, un mode de rapport et non l'absence de rapport.

S'il demeure un rapport dans la séparation, c'est que la représentation est aussi reflet, image, imitation. Nous avons l'habitude d'assimiler imitation et représentation; pourtant le concept d'imitation ou de mimésis est plus indéterminé - soyons exacts : il est historialement indéterminé - tandis que la représentation désigne un mode, le mode mimétique spécifiquement moderne. Imiter veut dire que l'identité se présente dans l'altérité, A se présente en $B$, le visage se présente en son portrait. Avant d'être une pratique visuelle, la mimésis est une donnée fondamentale de l'être en tant qu'être c'est-à-dire aussi bien en tant que paraître (c'est bien pourquoi il a fallu attendre une 
phénoménologie pour que la question de l'être se repose). Or si un des acquis heideggeriens est d'avoir montré que la question de l'être se noue à la question du temps de l'histoire, c'est aussi que les figures de l'être ou de la mimésis définissent des époques précises. Notre thèse serait donc la suivante : il n'y a pas "la" mimésis mais toujours des façons époquales d'imiter et de nouer le Même à l'Autre. La représentation est une de ces façons, elle est une figure de l'être (comme dit Heidegger) et une figure de l'être-politique (comme il ne le dit pas). Elle n'est probablement pas un concept et c'est pourquoi elle ne se laisse pas définir ; la ligne qu'elle dessine, du moins, peut être suivie: comme séparation. Nous appellerons figure le statut anté-conceptuel de la représentation dans la mesure où elle dessine et discrétise l'ouverture à l'être, où elle conditionne la mimésis et configure l'union politique. C'est en deçà du concept qu'il faut chercher le sens et l'unité de la représentation ${ }^{24}$.

Si chaque époque se définit par une figure, alors il faut ajouter que le moment cartésiano-hobbesien consiste à avoir substitué la figure de la représentation à celle de l'incarnation. C'est dans le tracé de l'incarnation en effet qu'une théorie du "corps politique" a pu être possible. La représentation n'a pu s'instituer qu'en invalidant sous telle ou telle de ses déterminations fondamentales la figure de l'incarnation, que ce soit en visant l'idée d'un corpus mysticum ecclesiae en 1642, ou en constituant une physique et une métaphysique qui évident le pilier eucharistique (ce qui est encore plus frappant chez Descartes qui, bien malgré lui, ne parvient pas à le sauver), ou encore dans la réinterprétation de la Trinité chez Hobbes (chacune des trois personnes divines "représente" Dieu; ce point est décisif pour la compréhension du passage de l'incarnation à la représentation, nous nous contentons ici d'y faire allusion).

Le couple incarnation/représentation permet de comprendre à la fois l'instauration de l'époque moderne et la portée de ce qui a été historiquement (donc définitivement) perdu parce qu'originairement configuré par l'incarnation. Au titre de figure, celle-ci ne se définit pas plus que la représentation, mais elle trace elle aussi un dessin que l'on peut parcourir. Nous pouvons décrire cette figure comme celle de l'inclusion réciproque. Selon ce schème, A est dans B et B est dans A. C'est chez Jean, c'est-à-dire, dans les tout derniers textes néotestamentaires - au moment où l'Ecriture devient théologie - que l'on trouve la version princeps de cette figure :

«Depuis si longtemps je suis avec vous et tu ne me connais pas, Philippe? Qui m'a vu a vu le Père. Comment peux-tu dire : montre-nous le Père! Ne crois-tu pas que je suis dans le Père et que le Père est en moi ? Les paroles que je vous dis, je ne les dis pas de moi-même, et c'est le Père demeurant en moi qui accomplit ses œuvres. Croyez-m'en : je suis dans le Père et le Père est en moi ; sinon, croyez à cause de mes œuvres. $»^{25}$

29 Le Fils incarné est le fils qui demeure en son père divin mais qui existentiellement (personaliter sed non substantialiter) sort de son père en le portant plus avant, en son sein propre. Cette inclusion mutuelle va dessiner jusqu'à l'aube de l'âge classique les termes les plus fondamentaux de la pensée, de la théologie, de la politique et de l'esthétique. Nous extrayons ici de la façon la plus raide quelques étapes majeures de l'époque incarnationnelle et de ses formules-clés, étant entendu que d'autres textes exigeraient aussi d'être évoqués dans une analyse beaucoup plus ample :

30 a) L'institution de l'eucharistie - Sacrement des Sacrements et perpétuation de la nouvelle alliance (semel et semper) - se formule ainsi : «Qui mange ma chair et boit mon sang demeure en moi et moi en lui » (Jean, VI, 56). 
31 b) L'institution de l'Eglise (après, certes la grande décision paulinienne) : «Quiconque confesse que Jésus est le fils de Dieu, Dieu demeure en lui, et lui en Dieu » (Première Epitre de Jean, IV, 15).

c) L'institution pontificale, chez Cyprien, au III siècle, garante de l'unité du corps mystique de l'Eglise : "L'évêque est dans l'Eglise et l'Eglise est dans l'évêque » (Lettre 66). L'autorité du pouvoir papal en est le fruit direct.

d) L'institution de l'icône comme image rédemptrice. Le texte fondateur se trouve chez saint Athanase, il sera repris à la fin de l'Apologie des saintes icônes de Jean Damascène lors de la querelle des images: "Et nous pouvons comprendre cela tout de suite en prenant pour illustration le portrait de l'empereur. Car dans le portrait, on trouve la figure et la forme de l'Empereur, et dans l'Empereur se trouve cette figure qui est dans le portrait. Car la similitude de l'Empereur et de l'image est exacte ; par conséquent qui regarde l'image voit l'Empereur, et qui voit l'Empereur, reconnaît aussi que c'est lui qui est dans le portrait. Et du fait que la ressemblance du portrait ne porte aucune différence, à celui qui souhaite voir l'Empereur d'après le portrait, celui-ci pourrait dire : "moi et l'Empereur sommes uns, car je suis en lui et il est en moi, et ce que tu vois en moi tu le perçois en lui, et ce que tu as vu en lui, tu le regardes en moi". Alors qui vénère l'image, en elle vénère l'Empereur aussi, car le portrait est sa forme et son apparence. Puisque le Fils aussi est l'Image du Père, il faut nécessairement comprendre que la divinité et la propriété du Père est l'essence du Fils » (Troisième discours contre les ariens, XXIII, 5). L'image incarnée prend sa part à l'économie divine, et s'allie à une dispensation du pouvoir, raison pour laquelle ce passage sera également signalé dans le cadre de la théologie politique.

e) L'institution du corps politique : on trouve par exemple, citée par Kantorowicz, cette formule de Lucas de Penna (vers 1300) : "Tout comme l'Eglise est dans le prélat et le prélat est dans l'Eglise, de même le Prince est dans la respublica et la respublica est dans le Prince ${ }^{26}$.

A chaque fois, cela "fait corps" ; mais une telle expression est encore insuffisante et il faudrait dire "cela (se) fait chair » pour rendre justice de ce nœud du tout et de sa partie capitale, de leur production au cœur du visible et de leur prétention à dire la vérité sur le juste et l'injuste. Il est donc légitime de considérer l'incarnation comme un régime historial qui aura irrigué et conformé la theoria, la praxis et la poiesis. Cette époque finit pourtant avec Descartes et Hobbes qui destituent cette figure pour dessiner la représentation. Il n'y a depuis le XVII ${ }^{\mathrm{e}}$ siècle plus de chair ou d'efficacité eucharistique ou de fidélité politique ; si ce n'est comme opinion religieuse privée, ce qui témoignerait encore du repli du religieux dans la conscience et donc de son alignement sur la représentation.

La distance s'est donc creusée entre la pensée hobbesienne de l'Etat et le schème corporatiste que figure le frontispice du Léviathan. Mais ce frontispice participe-t-il vraiment du régime incarnationnel? Si oui, pourquoi n'apparaît-il qu'à la tête de l'ouvrage qui le renverse? Des siècles de pensée icono-théologico-politique n'auront pas su produire de figure similaire, il est donc étonnant que ce soit de façon posthume que le "corps politique" ait trouvé la voie de sa représentation. Si non, quel sens (ou quel impact) politique a-t-elle donc? Le paradoxe doit être posé dans toute son acuité pour qu'on puisse commencer à répondre à ces questions. En apparence (et on ne pourra que s'interroger sur les modalités de cette apparence), le frontispice fait écho à la doctrine du "corps politique" (mais aussi à celle de la plenitudo potestas et encore à la 
théorie des deux glaives, tout en les convertissant à son profit ${ }^{27}$; ce mimétisme ironique est aussi un trait essentiel de la gravure de Hobbes/Bosse); et pourtant il ouvre sur une pensée de la représentation politique (il n'est précisément pas une représentation du politique).

L'unique point de départ qui se livre à nous pour étudier ce paradoxe, c'est donc le frontispice, ou plutôt sa force et ses effets, sa puissance de choc, voire sa célébrité et son caractère d'effigie diabolique. La réputation de cette image n'est pas un phénomène empirique étranger à sa nature, elle ne se surajoute pas au sens intime qu'elle délivre, jusque dans ses égarements et ses excès : la surinterprétation ou les mésinterprétations dont elle est l'objet lui appartiennent en propre. Nous nous prendrions nous-même à l'attraction impressionnante du frontispice si nous ne remettions pas en question le fait qu'elle énonce quelque vérité politique : peut-être l'image est-elle fausse. Détaillons donc non pas l'iconographie mais la disposition phénoménale du frontispice : il y a le colosse et il y a le titre (Léviathan). Le titre complet pose une identité entre le Léviathan et l'Etat, tandis qu'une citation de Job, XLI, 24 au-dessus du colosse parait poser la seconde équation entre le géant et le Léviathan; de là, transitivement semble établi le fait que le grand homme figure l'Etat. Et pourtant : cette série d'identifications relève elle aussi de la loi de la condensation. Or la condensation abuse, il est de sa nature d'abuser, de rapprocher des choses distinctes et de fusionner des entités singulières. Il ne s'agit pas de dire que l'image va simplement trop loin et de refuser tout aussi simplement les liens d'identification qu'elle noue, mais de reconnaître la loi qui la gouverne et qui est une loi de l'excès. En tant qu'image excessive ou excédante, le frontispice n'est peutêtre pas une représentation. Cette hypothèse n'a rien de gratuit et peut être étayée par quelques remarques sur le mot de Léviathan, qui est, notons-le, le surnom de l'Etat, et non pas son nom. Ce mot n'apparaît qu'à quatre reprises dans le livre :

38 a) Dans le titre. C'est alors le nom propre du livre, mais c'est aussi un nom qui se voit et même qui s'affiche. Léviathan, on va le constater, est dans une logique non pas seulement du visible mais de l'exhibition.

b) Dans le passage-clé de la fondation de l'Etat (chapitre XVII) : «Cela fait, la multitude ainsi unie en une seule personne est appelée RÉPUBLIQUE, en latin CIVITAS. Telle est la génération de ce grand Léviathan, ou plutôt pour en parler avec plus de révérence, de ce dieu mortel, auquel nous devons, sous le Dieu immortel, notre paix et notre protection » (nous soulignons). L'impudence comme abus de langage et démonstration de parade : à peine posé les termes propres de l'Etat, le Léviathan survient comme le "pas de trop".

40 c) Enfin, à deux reprises, dans une justification (chapitre XXVIII) : "Jusqu'ici, j'ai montré la nature de l'homme, que son orgueil et ses autres passions ont contraint de se soumettre à un gouvernement, ainsi que le grand pouvoir de celui qui le gouverne, que j'ai comparé à Léviathan, tirant cette comparaison des deux derniers versets du $41^{\text {ème }}$ chapitre du livre de Job: en cet endroit, Dieu, après avoir montré le grand pouvoir de Léviathan, l'appelle le roi des orgueilleux : il n'y a rien sur terre, dit-il qui puisse lui être comparé. Il est fait de telle sorte que rien ne peut l'effrayer. Toute chose élevée, il la voit audessous de lui. Il est le roi de tous les enfants de l'orgueil. »

41 Les occurrences du Léviathan le désigne comme l'exacerbation outrancière de l'Etat, son enflure orgueilleuse. On peut suivre le mouvement de cette exaltation dans la phrase de Hobbes qui dit comparer l'Etat à ce qui ne se compare à rien. Ce paradoxe montre le passage à la limite ou même le franchissement de la limite que signifie le 
Léviathan ; il n'appartient plus à aucune mimésis, il est la possibilité même de sortir du régime mimétique qui est celui de la représentation. C'est en ce sens qu'il s'expulse tout autant du champ théorique ou scientifique. Dans le De Corpore, Hobbes dit : « est objet de philosophie, tout corps dont il y a comparaison possible». La citation de Job qui surplombe le colosse du frontispice déplace ainsi le Léviathan hors de la représentation (métaphysique, picturale et politique) pour le transformer en une sorte d'idole qui concentre ou condense la puissance mais qui ne représente rien ni personne. Ce caractère d'idole ${ }^{28}$ se découvre jusque dans son iconographie ${ }^{29}$, nous pensons en particulier à la posture frontale du visage de Léviathan, tout à fait inusité, les portraits - réels, imaginaires ou allégoriques - évitant systématiquement le face à face. La triade frontalité-frontispice-effronterie décrit le Léviathan non comme l'Etat représentatif mais au contraire comme le franchissement de la représentation dans le mythe congelé et terrible de l'Etat. Cet excès demeure une possibilité du régime de la représentation et Hobbes indique clairement que l'Etat et le Léviathan sont co-originaires, ce qui a pour signification concrète que ce que l'on dénomme (dénomination problématique que nous nous contentons de suivre ici) démocratie produit le risque de son propre débordement, sous les modes historiquement et axiologiquement différents de la tyrannie, du despotisme, de la dictature ou du totalitarisme ${ }^{30}$. A chaque fois que cela s'est produit, c'est le Grand Corps politique qui a été rejoué, non plus comme autrefois de façon régulière, alignée sur l'incarnation, mais à contre-temps et à contre-histoire, (c'est-à-dire aussi en s'interrogeant sur la façon de faire l'histoire : sur la répétition historique, l'invention historique, la nostalgie historique ou le déni historique).

Il y aurait alors trois stades et non pas seulement deux, chez Hobbes : l'état de nature, l'Etat politique et le Léviathan. On peut leur faire correspondre, pensons-nous, trois affects : la peur, la "terreur" (terror) et la terreur panique. La distribution des deux premiers est clairement répétée par Hobbes lui-même ; il n'est pas inutile d'insister sur le fait que la "terreur" hobbesienne est positivement marquée. Si la peur disloque et altère (telle est « la peur au ventre»), la terreur, elle, est constructive car elle ne se vivifie qu'à l'approche de la transgression, dégageant ainsi dans l'espace des conduites possibles tout une étendue de quiétude et de sécurité (telle est la «peur du gendarme »). Quant à la terreur panique, elle nous paraît conforme à l'impressionnante puissance du Léviathan. Dans un passage de l'ouvrage, Hobbes décrit cet affect pour lui donner une signification politique particulière :

«La peur sans savoir pourquoi et de quoi est la TERREUR PANIQUE. Les mots viennent des fables qui font du dieu Pan leur auteur. A vrai dire, le premier qui est sujet à une telle peur a toujours la perception de ce qui la cause, bien que les autres prennent la fuite en suivant son exemple, chacun supposant que son voisin sait pourquoi. Et donc, personne n'éprouve cette passion s'il n'est dans une foule de gens ou dans une multitude. $»^{31}$

La foule ou la multitude n'est pas le peuple ${ }^{32}$, elle est en-deçà ou au-delà de la constitution sociale et politique, en-deçà lorsqu'à l'état de nature, les hommes sont dispersés, au-delà lorsqu'ils sont amassés dans un tout. C'est exactement ce que l'on voit dans le corps du Léviathan : non pas un peuple mais un conglomérat d'individus sans interstices ni liens. A ce titre le présent développement trouverait une pertinence particulière (mais non exclusive) dans le phénomène des masses modernes et du totalitarisme. Dans les régimes totalitaires, en effet, la dissolution des relations sociales les plus primaires se conjugue avec la formation idolâtre de l'incorporation totale; quant à la panique, on la reconnaîtrait sans difficulté non seulement, en masse, chez les 
victimes potentielles, mais encore dans la paranoïa politique des organes institutionnels et dans leur entreprise affolée d'expulsion des éléments étrangers hors du corps social. En 1921, soit peu de temps avant l'émergence des deux grandes formes de totalitarismes, ce lien entre la foule, la terreur panique comme sa possibilité la plus propre et l'image du grand corps, a fait l'objet du texte de Freud, Psychologie des foules et analyse $\mathrm{du}$ Moi. Freud y entreprend de mettre à découvert le fondement du lien social plus profondément que ne le font les notions d'instinct grégaire ou de suggestion, c'està-dire en faisant l'hypothèse d'un investissement libidinal. Cette hypothèse le mènera à une conception de la foule comme grand “corps", ce qui n'apparaît qu'au terme d'une analyse qui aura pris pour objet, les exemples "les plus intéressants" de l'Eglise (corpus Christi) et de l'Armée (corps militaire). Que ce soit ces deux formations qui aient exemplifié la foule dans son analyse psychologique est remarquable, car elles ne fondent aucune socialité à elles seules, au sens de la société civile: on voit combien c'est au-delà du politique qu'il faut aller chercher maintenant pour faire corps, comme on peut suspecter combien le politique n'est jamais débarrassé d'une telle possibilité. L'extrême coalescence des individus dans ces masses, infusée de libido, est à la hauteur de la panique qui survient lorsque le corps est décapité, ou même lorsque la rumeur que la tête est tombée circule :

«Le cas instructif et utile à nos fins, c'est celui ci-dessus mentionné d'un corps militaire ?Heereskörper? pris de panique alors que le danger n'a pas dépassé la mesure habituelle, fréquemment bien supportée. (...) La cause typique de l'irruption d'une panique est tout à fait semblable à celle qui est représentée dans la parodie que Nestroy fait du drame de Hebbel, Judith et Holopherne. Un guerrier s'y écrie : "Le général a perdu la tête", et là-dessus tous les Assyriens prennent la fuite. La perte du meneur, de quelque manière qu'on l'entende, la perplexité dont il est l'objet, font surgir la panique, alors que le danger reste le même; avec le lien au meneur disparaissent aussi - en règle générale - les liens mutuels des individus de la foule. La foule se pulvérise comme un flacon de Bologne dont on a coupé la pointe. $»^{33}$

Si la panique peut survenir à l'occasion de la décapitation du corps, c'est que celui-ci ne faisait que la contenir, exactement comme le flacon ne faisait que renfermer le parfum qui se pulvérisera et exactement comme le corps du Léviathan ne fait que contenir les homuncules sans les constituer politiquement. Le choix de l'Eglise et de l'armée montre quelle transformation doit affecter l'Etat pour qu'il puisse devenir un "corps" et ce faisant être sujet à la panique. La représentation modère heureusement l'amour politique; elle délie et sépare. Jamais complètement sans doute. Mais lorsque l'investissement libidinal surpasse l'investiture d'un souverain, le "pas de trop" est franchi. La communauté rejoue alors le jeu du "grand corps", mais de manière fantasmée, avec toute l'efficacité du fantasme, qui est avant tout fantasme du corps sacré et guerrier. On a souvent décrit le frontispice du Léviathan comme ordonnant les deux pouvoirs spirituel et temporel, mais regardons-y bien : il s'agit bien plutôt des attributs ecclésiastiques et militaires (et non pas civils : une forteresse, un canon, une batterie de fusils et un champ de bataille).

"Qu'on s'imagine un corps plein de membres pensants! », disait Pascal ${ }^{34}$. A la naissance de notre modernité, qui a vidé la figure incarnationnelle de sa sève, il reste un lieu où un grand corps est possible : c'est celui - sans garantie - de la morale chrétienne. Elle est désormais sans garantie parce qu'elle est en deuil de sa source ; et pour cette raison elle est un pari. Or on ne gage pas la paix civile; la morale chrétienne se retire ainsi d'elle-même du champ politique ${ }^{35}$. Le frontispice du Léviathan est le foyer exalté d'une 
mystique politique dans laquelle l'Etat moderne risque congénitalement de verser. En cela, il nous fascine, en cela il peut aussi nous prévenir : on n'entre pas en politique sans courir ce risque-là.

\section{NOTES}

1. Hobbes, Léviathan ou matière, forme et puissance de l'Etat chrétien et civil, trad. G. Mairet, Paris, Gallimard, 2000, p. 351 : «la souveraineté est l'âme de l'Etat: une fois séparée du corps, les membres ne reçoivent plus d'elle leur mouvement ».

2. Ainsi l'étranger, à Rome, doit-il être peu à peu traité juridiquement "comme s" il était un citoyen; d'où la multiplication de procédures fictives et pourtant effectives. L'origine du droit étant intouchable et irréversible, il reste à celui-ci, pour se développer, d'élaborer des fictions juridiques.

3. N. Malcolm, "The Titlepage of Léviathan, Seen in a Curious perspective ", The Seventeenth Century, 13.2 (automne 1998), pp. 124-155 ; H. Bredekamp, Stratégies visuelles de Thomas Hobbes, trad. Denise Modigliani, Paris, MSH, 2003.

4. J.-F. Nicéron, La Perspective curieuse, Paris, 1638, p. 115.

5. "The Answer to Sir Will. Davenant's Preface Before Gondibert ", inSir William Davenant's "Gondibert ", éd. D. F. Gladish, p. 55.

6. Préface à la traduction anglaise du Pastor Fido de Guarini, 1648, cité et traduit in H. Bredekamp, op. cit. p. 88. Malcolm, art. cit. p. 128.

7. Ibid. p. 87. Nombreuses autres formulations aussi définitives passim..

8. A un détail près, qui n'est pas mince : le frontispice n'est pas une anamorphose et ne nécessité aucun dispositif polyoptrique pour être vu. Cela n'empêche pas H. Bredekamp d'affirmer que «l'éclatement des partis ennemis lui (à Hobbes) suggère l'image anamorphotique du Léviathan, dont il fait précéder, en frontispice, son œuvre propre », op. cit. p. 90.

9. République, VIII, 556e sqq..

10. Cela concernerait encore Aristote (Politiques, 1253a20-25 ; Mouvement des animaux, 703a30), la tradition cosmopolitique chez les Stoïciens (Cicéron, De Officiis, III, v, 22 ; Sénèque, De ira, II, 31, De clementia, I, v, 1 ; Epictète, Entretiens, II, 10) ou Menenius Agrippa s'adressant à la plèbe (Tite-Live, II, XXXII).

11. Il faut noter que si l'expression de «théologie politique » a été initialement élaborée par Carl Schmitt, celui-ci l'identifiait non pas à l'époque médiévale-scolastique (pour dire les choses trop simplement) mais à l'époque moderne, celle qui commence avec Hobbes. C'est là un autre problème, qui concerne Schmitt lui-même. Kantorowicz est responsable du déplacement ou du replacement de la théologie politique comme politique chrétienne médiévale.

12. Elements de la loi naturelle et politique, trad. D. Weber, Paris, Librairie Général Française, 2003, p. 223.

13. «Représentation et communauté - Sur Thomas Hobbes », Archives de philosophie, (à paraître).

14. Parmi d'autres occurrences: «selon les deux principales parties de l'homme, je divise ses facultés en deux espèces : facultés du corps et facultés de l'esprit », Elements, op. cit. p. 81.

15. De Cive XVII, xxii. 
16. La différence est de taille, elle permet notamment de spécifier les parties spirituelles de cet homme artificiel (mémoire, raison, volonté, parole et bien sûr l'âme) ce que n'a pu faire le théorie $\mathrm{du}$ "corps politique".

17. Op. Cit. p. 64. Une seule autre exception, strictement rhétorique (il s'agit de la métaphore de l'épilepsie appliquée à l'Etat, qui pousse à le comparer à un corps), p. 487.

18. Op. cit. p. 295.

19. Pour deux raisons distinctes : d'une part le souverain représente le peuple en tout, d'autre part nul n'échappe à la représentation: celui qui n'est pas le représentant d'un autre se représente lui-même (op. cit. p. 271).

20. "L'état d'hostilité et de guerre étant tel que la nature elle-même, par lui, est détruite ", Elements de la loi naturelle et politique, I, xiv, op. cit. p. 181.

21. La décision métaphysique de Hobbes, Paris, Vrin, 1987, pp. 36-58.

22. Eléments de la loi naturelle et politique, op. cit. p. 82.

23. Tractatus Opticus (1640), Secunda Sectio (vers 1644), A Minute or First Draughtof the Optiques (1646), De Homine (1658).

24. Kant, ici, serait d'un intérêt tout particulier (et ce n'est pas un hasard, à défaut d'être juste, si le premier kantien, Karl Leonhard Reinhold, a cru pouvoir fonder l'intégralité du kantisme sur une Vorstellungstheorie; il voit juste en admettant la représentation comme pivot de sa pensée, il se trompe en incluant celle-ci dans l'ordre de la logique et du concept - qu'il ne parvient d'ailleurs jamais à définir réellement). La Logique (introduction, V) insiste sur le caractère irréductible et indéfinissable de la Vorstellung, et l'opsucule Vers la paix perpétuelle (premier article définitif) déclare que toute constitution qui n'est pas repräsentativ est proprement une Unform. Voilà qui reconnaît assez le sens figural de la représentation.

25. Jean, XIV, 8-11.

26. Kantorowicz, Les deux corps du roi, trad. J.-Ph. Genet et N. Genet, Paris, Gallimard, coll. Quarto, 2000, p. 954. De nombreuses formules similaires parcourent le Moyen Age, jusqu'au XVI ${ }^{\mathrm{e}}$ siècle.

27. Ce que l'augustinisme politique attribue à la souveraineté pontificale - souveraineté absolue et contrôle du séculier comme du spirituel - l'image l'attribue au souverain politique. La loi de la condensation régit, plus que celle de l'incorporation, l'iconographie du frontispice.

28. On pourrait comparer le buste de Léviathan avec les gravures plus ou moins fidèles d'idoles orientales à la mode vers 1650 ; voir en particulier la proximité étonnante avec l'Isis multimammia dont on trouvera des illustrations dans Baltrušaitis, La quête d'Isis, Paris, Flammarion, 1985, p. 137.

29. Il y aurait à développer la question des rapprochements iconographiques qui servent à étayer une thèse herméneutique ; plusieurs généalogies étant à chaque fois possibles, qu'est-ce qui détermine une filiation plutôt qu'une autre?

30. Soyons précis : nous ne prétendons pas ici expliquer l'une ou l'autre de ces condensations politiques, ni les placer au même niveau. Il s'agit uniquement de viser la condition qui les a rendus possibles.

31. Léviathan, op. cit p. 132.

32. De Cive, trad. Sorbière, éd. S. Goyard-Fabre, Paris, Flammarion, 1982, p. 149.

33. Psychologie des foules et analyse du Moi, trad. coll., Paris, Payot, 1985, p. 159.

34. Pensées, édition Ph. Sellier, fragment 403. Toute la section intitulée "Morale chrétienne » fonctionne sur cette figure.

35. «Deux lois suffisent pour régler toute la république chrétienne mieux que toutes les lois politiques », ibid. fr. 408 . 
INDEX

Mots-clés : Frontispice, Hobbes, Léviathan 